\title{
Influence of Upper Extremity Muscle Mass on Lateral Pinch Strength
}

\section{Daisuke Takagi}

Department of Physical Therapy, Health Science University, 7187 Kodachi Fujikawaguchiko-Town, Yamanashi, 401-0380, Japan

\section{Abstract}

Background: Pinch strength is required for activities of daily living, and any decline in it can cause difficulty with fine motor skills, such as fastening buttons or tying shoelaces. Thus, it is important to prevent the deterioration of pinch strength. Muscle strength is positively related to muscle mass. Conversely, it has been reported that changes in muscle mass and muscle strength do not always correspond and bioelectrical impedance analysis is a relatively easy method to measure muscle mass. But there has been little study of how upper extremity muscle mass using bioelectrical impedance analysis influences pinch strength. In this study, we aimed to investigate the relationship between upper extremity muscle mass and pinch strength. Furthermore, we examined the association between upper extremity fat mass and pinch strength.

Methods: This cross-sectional study included 82 healthy young men and women aged 20-22 years (mean age, $21.1 \pm 0.7$ years). Maximum lateral pinch strength was measured using a digital pinch sensor. Upper extremity muscle mass and fat mass were estimated using bioelectrical impedance analysis. The relationships between pinch strength, upper extremity muscle mass, and upper extremity fat mass were assessed using correlation and multiple regression analyses.

Results: Men had a greater pinch strength and upper extremity muscle mass than women $(\mathrm{P}<0.05)$; however, there was no significant difference in upper extremity fat mass $(\mathrm{P}>0.05)$. Pinch strength showed a significantly positive association with upper extremity muscle mass $(\mathrm{P}<0.05)$, but not with upper extremity fat mass $(\mathrm{P}>0.05)$. In multiple regression analysis adjusted for sex, upper extremity muscle mass was found to be a significant explanatory variable for pinch strength $(\mathrm{P}<0.05)$.

Conclusion: To prevent the decline in pinch strength, preserving the ability of daily living activities that involve the hands, such as fastening buttons or tying shoelaces, needs to be focused on by improving upper extremity muscle mass using bioelectrical impedance analysis.

\section{Introduction}

The proportion of people aged 65 years or older has been increasing worldwide, and the Cabinet Office, Government of Japan has estimated that by $2065,38.4 \%$ of the total population of Japan will be aged above 65 years [1]. Aging is associated with a decline in physical function and, in some cases, onset of frailty, a condition of enhanced vulnerability to stressors that can result in adverse health outcomes and dependency on others [2,3]. Therefore, preventing the deterioration in the ability to perform activities of daily living (ADL) to extend healthy life expectancy of elderly people is of substantial benefit.

A deterioration of muscle strength can lead to disability and dependency [3]. Previous studies have typically focused on the aspects of maintaining or improving an individual's ability to perform ADLs related to the muscle strength of the lower extremities (such as gait or climbing stairs), which are commonly affected by aging. However, elderly people may also need assistance with ADLs that involve their hands or upper extremities, including those requiring fine motor skills. Pinch strength (PS) is associated with the ability to perform ADLs, and a decline in PS can make it difficult for individuals to perform tasks that require fine motor skills, such as fastening buttons or tying shoelaces [4,5,6]. PS decreases in old age [4], and it is important to prevent this deterioration to preserve an individual's fine motor ability which is one of the factors that affect ADLs.

Muscle strength is positively related to muscular cross-sectional area and muscle mass $[7,8]$. Conversely, it has been reported that changes

\section{Publication History:}

Received: April 02, 2019

Accepted: April 30, 2019

Published: May 02, 2019

\section{Keywords:}

Hand force, Body composition, Bioelectrical impedance analysis in muscle mass and muscle strength do not always correspond [9]. To measure muscle mass using bioelectrical impedance analysis (BIA) is relatively easy and inexpensive compared with other methods, such as computed tomography (CT), magnetic resonance imaging (MRI), and dual-energy X-ray absorptiometry (DXA). However, there has been little investigation of how upper extremity muscle mass (UMM) using BIA influences PS. Clarification of the association between UMM and PS could be useful for suggesting the need to focus on UMM for maintaining or improving PS, especially in elderly; this might eventually lead the maintenance of an individual's fine motor skills and independence in ADLs and make it possible to evaluate muscle mass more easily.

The aim of this study was first to investigate the relationship between UMM using BIA and PS in healthy young men and women with less impact of muscle atrophy by disease. In addition, we examined the association between upper extremity fat mass (UFM) and PS.

*Corresponding Author: Dr. Daisuke Takagi, Department of Physical Therapy Health Science University: 7187 Kodachi Fujikawaguchiko-Town, Yamanashi, 401-0380, Japan. Tel: +81 555-83-5295; Fax: +81 555-83-5299; E-mail: pt.takadai@gmail.com

Citation: Takagi D (2019) Influence of Upper Extremity Muscle Mass on Lateral Pinch Strength. Int J Phys Ther Rehab 5: 150. doi: https://doi.org/10.15344/2455$7498 / 2019 / 150$

Copyright: (C) 2019 Takagi. This is an open-access article distributed under the terms of the Creative Commons Attribution License, which permits unrestricted use, distribution, and reproduction in any medium, provided the original author and source are credited. 


\section{Methods}

\section{Participants}

This cross-sectional study initially included 85 healthy young men and women aged 20-22 years. Two exclusion criteria were applied: anyone with a pacemaker and/or metal implant placed by surgery; and anyone who had undertaken $\geq 30$ minutes of physical exercise regularly at least twice a week for the previous year [10]. Two people were excluded because of having a metal implant and one because of an exercise habit. Thus, 82 participants ( 48 men and 34 women) were included in the analysis. Of them, $74(90 \%)$ participants showed right hand dominance; 6 men and 2 women were left-handed. The study was approved by the ethics committee of Health Science University, and all the participants read and signed an informed consent form.

\section{Pinch strength}

Maximum lateral PS was measured three times on each side with a digital pinch sensor (Isoforce GT-300, OG GIKEN, Okayama, Japan). The output range of the pinch force was 0.3-10.2 kgf. The method was as described in previous reports [11,12]: with the participant in a standing position, the measurements were made with the shoulder adducted, the elbow in $90^{\circ}$ flexion, and the forearm in a neutral position. The participant grasped the pinch sensor between the pulp of the thumb and the radial side of the distal interphalangeal joint of the index finger. The maximum left and right PS values were used as a representative value.

\section{Upper extremity muscle and fat mass}

The left and right UMM and the left and right UFM were estimated by segmental multifrequency BIA at 5, 50 and $250 \mathrm{~Hz}$ using an ioi353S system (Owa Corporation, Tokyo, Japan). BIA has been reported to provide a reliable and valid method for estimating both muscle and fat mass $[13,14]$. The participant stood barefoot on the device to measure his or her body weight. After the participant's age, sex, and height were entered into the system, the participant gripped the handles and positioned both palms and the soles of both feet so they were in constant contact with the electrodes for the estimation of UMM and UFM.

\section{Statistical analysis}

The data are presented as means \pm standard deviations. The statistical analyses were performed with JMP 11 software (SAS Institute Inc., Cary, NC, USA). Unpaired t-tests were used to evaluate differences in UMM, UFM, and PS between the sexes, and relationships between UMM, UFM, and PS were assessed by Pearson's correlation analysis. Where there were significant differences in PS between men and women, multiple regression analysis adjusted for sex was used to assess the influence of UMM or UFM on PS. Statistical significance was set at $\mathrm{P}<0.05$.

\section{Results}

Table1 presents a comparison of participant characteristics between the men and women. There were no significant differences in age or left and right UFM (all P > 0.05), whereas height, weight, body mass index, left and right UMM, and left and right PS were higher in the men than the women (all $\mathrm{P}<0.001$ ). Table 2 shows the results of the Pearson's correlation analyses. There was a significant positive correlation between left PS and left UMM $(r=072, P<0.001$; Figure 1A) but not left UFM ( $r=0.17, P=0.13$; Figure $1 B)$. Similarly, there was a significant positive correlation between right $\mathrm{PS}$ and right $\mathrm{UMM}$ $(\mathrm{r}=0.72, \mathrm{P}<0.001$; Figure 1C) but not right UFM $(\mathrm{r}=0.19, \mathrm{P}=0.08$, Figure 1D). Multiple regression analysis adjusted for sex showed that left and right UMM were statistically significant explanatory variables for left and right PS, respectively $(\mathrm{P}<0.05$; Table 3$)$.

\section{Discussion}

In this study, UMM but not UFM using BIA was positively related to PS in healthy men and women. UMM was also a statistically significant independent explanatory variable for PS in the multiple regression analysis adjusted for sex. These findings would help in recommending the need to focus on UMM for maintaining or improving PS, especially in elderly, thereby allowing an individual to retain their independence in performing ADLs that involve the fine motor skills of the hands, such as fastening buttons or tying shoelaces and make it possible to evaluate muscle mass more easily.

In this study, the mean values of right and left PS were 7.8 and 7.3 kgf in men and 5.4 and 5.0kgf in women, respectively. The mean values of right and left PS reported in a previous study of healthy Japanese subjects aged 21-39 years were higher than those in the present study: 11.5 and $10.7 \mathrm{~kg}$ for men and 7.9 and $7.6 \mathrm{~kg}$ for women, respectively [12]. According to Ministry of Health, Labour and Welfare in Japan

\begin{tabular}{|l|l|l|l|l|}
\hline Characteristics & Total $(\mathrm{N}=82)$ & Men $(\mathrm{n}=48)$ & Women $(\mathrm{n}=34)$ & P value \\
\hline Age $($ years $)$ & $21.1 \pm 0.7$ & $21.1 \pm 0.6$ & $21.0 \pm 0.7$ & 0.52 \\
\hline Height $(\mathrm{cm})$ & $165.7 \pm 8.7$ & $171.3 \pm 5.3$ & $157.8 \pm 6.0$ & $<0.001$ \\
\hline Weight $(\mathrm{kg})$ & $58.7 \pm 11.8$ & $65.5 \pm 10.3$ & $49.2 \pm 5.6$ & $<0.001$ \\
\hline BMI $\left(\mathrm{kg} / \mathrm{m}^{2}\right)$ & $21.2 \pm 3.0$ & $22.3 \pm 3.2$ & $19.7 \pm 1.8$ & $<0.001$ \\
\hline Left PS $(\mathrm{kgf})$ & $6.4 \pm 1.5$ & $7.3 \pm 1.0$ & $5.0 \pm 0.9$ & $<0.001$ \\
\hline Right PS $(\mathrm{kgf})$ & $6.8 \pm 1.7$ & $7.8 \pm 1.2$ & $5.4 \pm 1.2$ & $<0.001$ \\
\hline Left UMM $(\mathrm{kg})$ & $2.83 \pm 0.59$ & $3.23 \pm 0.39$ & $2.26 \pm 0.25$ & $<0.001$ \\
\hline Right UMM $(\mathrm{kg})$ & $2.84 \pm 0.59$ & $3.24 \pm 0.39$ & $2.27 \pm 0.27$ & $<0.001$ \\
\hline Left UFM $(\mathrm{kg})$ & $0.73 \pm 0.28$ & $0.74 \pm 0.34$ & $0.71 \pm 0.16$ & 0.58 \\
\hline Right UFM $(\mathrm{kg})$ & $0.72 \pm 0.28$ & $0.73 \pm 0.34$ & $0.70 \pm 0.15$ & 0.54 \\
\hline
\end{tabular}

Table 1: Characteristics of the study participants.

Values are expressed as mean \pm standard deviation. BMI, body mass index; PS, pinch strength; UMM,upper extremity muscle mass; UFM, upper extremity fat mass 
Citation: Takagi D (2019) Influence of Upper Extremity Muscle Mass on Lateral Pinch Strength. Int J Phys Ther Rehab 5: 150. doi: https://doi.org/10.15344/2455$7498 / 2019 / 150$

(2015), the mean height and weight of Japanese people aged 20 years are $173.2 \mathrm{~cm}$ and $64.6 \mathrm{~kg}$ for men and $156.4 \mathrm{~cm}$ and $50.0 \mathrm{~kg}$ for women, respectively [15]; the mean height and weight of the participants in this study were consistent with those for the general population. The lower PS values in this study may therefore be the result of differences in equipment and methods, or a greater age range in the previous study. Further studies are needed to correspond with the methods of measurements and characteristics of subjects.

In this study, there was a significant positive association between UMM and PS. Muscle strength depends on the muscular crosssectional area and muscle mass $[7,8]$, so UMM might have an impact on PS. Conversely, the values for the standardized partial regression coefficients for the associations between left and right UMM and PS were 0.27 and 0.41 , respectively. The level of muscle exertion is affected not only by muscle mass but also by aspects of the nervous system (such as motor unit recruitment) [16], and there are variations between individuals in the relationship between muscular crosssectional area and muscle strength [17]. The standardized partial regression coefficients of UMM for PS were relatively low in this study. However, effects of the nervous system were not measured. Associations between the nervous system and muscle mass should be clarified in future studies.

\begin{tabular}{|l|l|l|l|l|l|}
\hline Variables & Left PS & P value & Variables & Right PS & P value \\
\hline Left UMM & 0.72 & $<0.001$ & Right UMM & 0.72 & $<0.001$ \\
\hline Left UFM & 0.17 & 0.13 & Right UFM & 0.19 & 0.08 \\
\hline
\end{tabular}

Table 2: Pearson coefficients for correlations between upper extremity muscle mass (UMM) or upper extremity fat mass (UFM) and pinch strength (PS) $(\mathrm{N}=82)$.

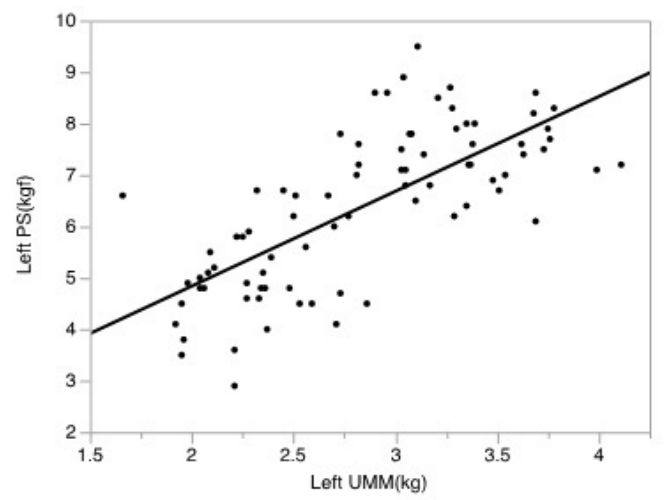

Figure 1A

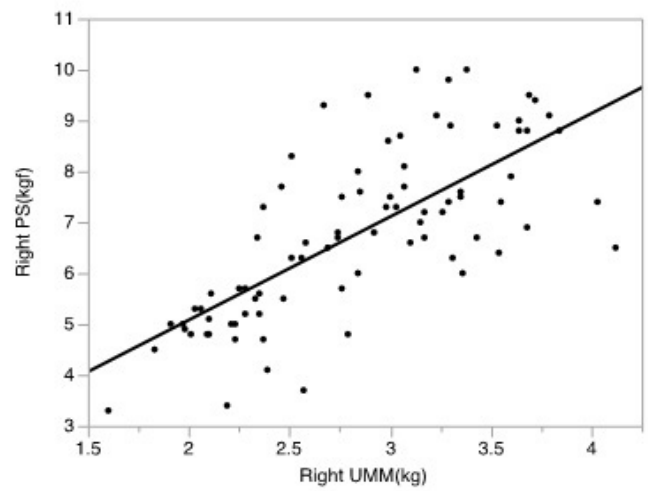

Figure 1C

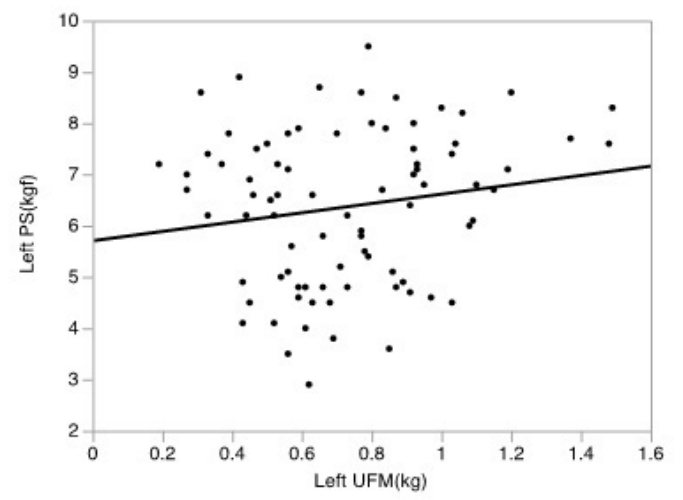

Figure 1B

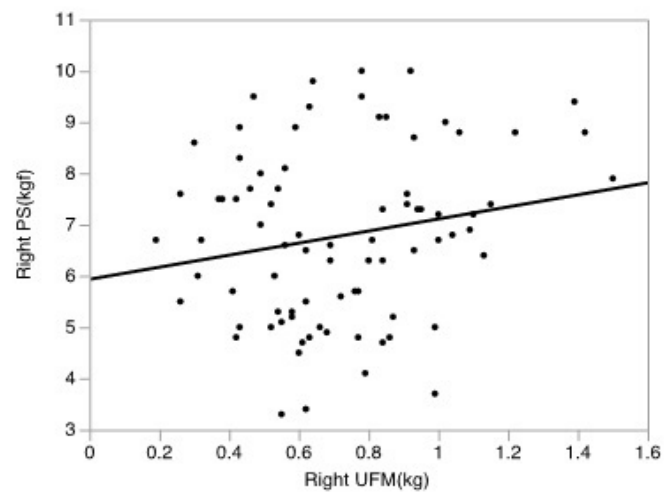

Figure 1D

Figure1A,1B,1C,1D: The association of pinch strength with upper extremity muscle and fat mass.

Left PS: Left pinch strength; Right PS: Right pinch strength; Left UMM: Left upper extremity muscle mass; Right UMM: Right upper extremity muscle mass; Left UFM: Left upper extremity fat mass; Right UFM: Right upper extremity fat mass.

\begin{tabular}{|l|l|l|l|l|l|}
\hline Variables & Left PS & P value & Variables & Right PS & P value \\
\hline Sex & 0.55 & $<0.001$ & Sex & 0.38 & 0.0036 \\
\hline Left UMM & 0.27 & 0.03 & Right UMM & 0.41 & 0.0016 \\
\hline
\end{tabular}

Table 3: Standardized partial regression coefficients for associations among pinch strength (PS), sex, and upper extremity muscle mass $(\mathrm{UMM})(\mathrm{N}=82)$. 
Sex was a significant independent explanatory variable for PS in the multiple regression analysis in the present study. Muscle strength is positively associated with muscular cross-sectional area and muscle mass $[7,8]$. On the other hand, muscle strength per muscular crosssectional area is not significantly different between the sexes [18]. In the present study, no significant differences in the average values of PS/UMM were observed in both left and right sides between the sexes [left (men vs women): $2.3 \pm 0.37$ vs $2.2 \pm 0.47$; right (men vs women): $2.4 \pm 0.41$ vs $2.4 \pm 0.40$, all $\mathrm{P}>0.05$ ]. PS in men was significantly higher compared with that in women and sex may be extracted as a significant independent explanatory variable for PS in multiple regression analysis. The standardized partial regression coefficient between PS and UMM in the multiple regression analysis adjusted for sex was also higher for the right side than the left side. Sex had a stronger impact on left PS in this study. Japanese women typically use both hands for ADLs, such as cooking and applying make-up. Muscle strength is affected by the nervous system [16] and there is not always a linear association between muscle strength and muscle mass [19]. This may explain the greater mismatch between PS and muscle mass in women than in men in this study.

This study had several limitations. The number of participants was small, with little variation in characteristics, and the study included only healthy men and women. Future studies with more participants and a wider range of characteristics (such as including communitydwelling elderly people) are needed. In addition, UMM and UFM were not localized to specific muscles in this study. Further studies are needed to elucidate the association between site-specific muscle mass and PS.

\section{Conclusions}

PS showed a significant positive association with UMM but not with UFM using BIA. UMM was also a significant independent explanatory variable for PS in multiple regression analysis. This finding could be useful in recommending the need to focus on UMM for maintaining or improving PS and make it possible to evaluate muscle mass more easily.

\section{Competing interests}

The author declares that they have no competing interests.

\section{References}

1. Cabinet Office, Government of Japan (2018) Situation on aging.

2. Inouye SK, Studenski S, Tinetti ME, Kuchel GA (2007) Geriatric syndromes: clinical, research, and policy implications of a core geriatric concept. J Am Geriatr Soc 55: 780-791.

3. Fried LP, Tangen CM, Walston J, Newman AB, Hirsch C, et al. (2001) Frailty in older adults: evidence for a phenotype. J Gerontol A BiolSci Med Sci 56: 146-156.

4. Jansen CW, Niebuhr BR, Coussirat DJ, Hawthorne D, Moreno L, et al. (2008) Hand force of men and women over 65 years of age as measured by maximum pinch and grip force. J Aging Phys Act 16: 24-41.

5. Bae JH, Kang SH, Seo KM, Kim DK, Shin HI, et al. (2015) Relationship Between Grip and Pinch Strength and Activities of Daily Living in Stroke Patients. Ann Rehabil Med 39: 752-762.

6. Grabiner MD, Enoka RM (1995) Changes in movement capabilities with aging. Exerc Sport Sci Rev 23: 65-104.

7. Koopman $\mathrm{R}$, van Loon $\mathrm{U}$ (2009) Aging, exercise, and muscle protein metabolism. J Appl Physiol 106: 2040-2048.
8. Rolland $Y$, Lauwers-Cances $V$, Cournot $M$, Nourhashémi $F$, Reynish $W$, et al. (2003) Sarcopenia, calf circumference, and physical function of elderly women: a cross-sectional study. J Am Geriatr Soc 51: 1120-1124.

9. Goodpaster BH, Park SW, Harris TB, Kritchevsky SB, Nevitt M, et al. (2006) The loss of skeletal muscle strength, mass, and quality in older adults: the health, aging and body composition study. J Gerontol A Biol Sci Med Sci 61: 1059-1064.

10. Ministry of Health, Labour and Welfare. Health Japan 21.

11. Mathiowetz V, Kashman N, Volland G, Weber K, Dowe M, et al. (1985) Grip and pinch strength: normative data for adults. Arch Phys Med Rehabil 66: 69-74.

12. Suzuki T, Kuniyoshi K, Matsudo T, Yamada T, Itadera E, et al. (2007) Evaluation of grip and pinch strength difference between dominant and non-dominant hand. J Jpn Soc Surg Hand. 24: 24-27.

13. Fornetti WC, Pivarnik JM, Foley JM, Fiechtner JJ (1999) Reliability and validity of body composition measures in female athletes. J Appl Physiol 87: 1114-1122.

14. Miyatani M, Kanehisa H, Masuo Y, Ito M, Fukunaga T, et al. (2001) Validity of estimating limb muscle volume by bioelectrical impedance. J Appl Physiol 91: 386-394.

15. Ministry of Health, Labour and Welfare, mean hight and body weight by sex and age by year.

16. Manini TM, Clark BC (2012) Dynapenia and aging: an update. J Gerontol A Biol Sci Med Sci 67: 28-40.

17. Yamada S, Fukunaga T (2003) Biochemical and physiological adaptations to physical training in skeletal muscle. NAP Limited, Japan, pp. 13-23.

18. Maughan RJ, Watson JS, Weir J (1983) Strength and cross-sectional area of human skeletal muscle. J Physiol 338: 37-49.

19. Cruz-Jentoft AJ, Baeyens JP, Bauer JM, Boirie Y, Cederholm T, et al (2010) Sarcopenia: European consensus on definition and diagnosis: Report of the European Working Group on Sarcopenia in Older People. Age Ageing 39: 412-423.
This article was originally published in a special issue: Various Approaches for Rehabilitation Science-Vol II Handled by Editor(s): 